\title{
Taiwan Mandarin - Does It Remain Homogeneous?
}

Huiju Hsu

hsuhj.hsu@msa.hinet.net

Department of English, National Taiwan Normal University

\begin{abstract}
Previous studies have shown discrepancies in tonal realizations between Guoyu and Putonghua. Early studies suggests Guoyu $\mathrm{T} 3$ is predominantly a falling tone and recent studies show Guoyu T2 is predominantly a dipping tone, contrastive to the long-considered default dipping and rising tone respectively. This study further explores the existence of regional varieties of Guoyu. Data are collected from Taipei and Taichung. Speakers read target sentences with 19 minimal pairs of final T2/T3 syllable being placed sentence final. Results indicate regional differences of T2/T3 patterns. The result of Taipei speakers indicates a clear distinction of $\mathrm{T} 2 / \mathrm{T} 3$ contour in that $\mathrm{T} 2$ is realized as a mid-dipping contour and T3 either a mid-dipping or a mid-falling contour, with the latter as the majority. However, in the Taichung dialect, this distinction disappeared. It is shown that Taichung T2 contour has changed from mid-dipping to mid-falling, merging with T3. This merger is statistically significant.
\end{abstract}

\section{INTRODUCTION}

Standard Mandarin, which was based on the Beijing dialect, was officially introduced into Taiwan mostly by non-native speakers of the dialect. For the past more than five decades, the contacts between Mandarin speakers in Taiwan and China have been limited, resulting in the appearance of a new version of Standard Mandarin in Taiwan, or Guoyu, literally meaning national language. Previous studies have observed some discrepancies between Guoyu and Putonghua. For example, Kubler [5] describes that instead of 214 in Putonghua as described by Chao [1], the tonal value of T3 in Guoyu is 21 or 31 . In other words, T3 of Taiwan Mandarin is a falling tone, as dipping does not occur in the tonal contour of Southern Min, the major local language in Taiwan. Lo [6] also suggests that T3 in Taiwan Mandarin is realized as either a low level tone or low falling tone, with tonal value of 11 and 21 respectively. Fon \& Chiang [3] acoustically analyzes the tonal value of Guoyu, and proposes the tonal contours of Taiwan Mandarin as follows: T1: 44;T2: 323; T3: 31(2); T4: 42. Note that T2 is realized as a dipping tone. Tzeng [9] conducts an experiment and acoustically analyzes Mandarin speech of news broadcasters in both Taiwan and China. The results also demonstrate that the tonal system of Guoyu covers a lower and narrower pitch range $(139-309 \mathrm{~Hz})$, while that of Putonghua covers a higher and wider pitch range $(146-381 \mathrm{~Hz})$. A recent study of Fon \& Hsu [2] further points out that the distinction between T2 and T3 in Guoyu seems to be in the process of neutralization.

In terms of language contact and language change, both historical linguists and linguistic variationists claim consistent changes of languages exist, not only diachronically but also synchronically [7]. Therefore, after its controversial implementation, it is reasonable to expect that the now localized Guoyu would show some signs of the next stage, diversification. This study thus plans to investigate such a phenomenon through some observations of the two nonhigh tones in the system, and hopefully to clarify the long-standing myth that Guoyun is homoegeneous. 


\section{METHOD}

\subsection{Subjects}

Four subjects from Taipei and Taichung, one male and one female in each city, were recruited in this study. All four subjects received post-graduate education. The subjects were Guoyu-Taiwan Min bilinguals. Bilinguality is loosely defined as that the subjects must learn these two languages natively, and that they can, on a self-report basis, fluently speak these two languages.

The age of the subjects ranged from 28-35. This age range was used to maximize the possibility of the subjects' nativeness of Taiwan Mandarin, as TV broadcasting (in Mandarin) began in 1962 in Taiwan and the 9-year compulsory education policy was initiated in the year 1968. Hsu [4] observes an apparent boundary of language shift from Southern Min to Mandarin in a four-generation family in Taiwan. This boundary corresponds to these two turning-point yearsof Mandarin promotion in Taiwan.

The two sampled regions are Taipei and Taichung. Taipei is the political center of Taiwan and, in terms of language use, is considered the center of Mandarin, the only official language in Taiwan. On the other hand, Taichung is selected as the sample region of the Mandarin-Southern Min bilingual region. Taichung is chosen due to two reasons. First, as Taipei, Taichung is also one of the major urban areas in Taiwan. Second, on the north-to-south west corridor of Taiwan, the major industrial areaon the island, Taichung is the first major Southern-Min/ Mandarin region south of Taipei.

\subsection{Stimuli}

19 minimal pairs of $\mathrm{XY}_{-\mathrm{T} 2}$ and $\mathrm{XY}^{-}{ }_{\mathrm{T} 3}$ were included in the study. The 38 tokens of the 19 minimal pairs were placed in semantically meaningful carrier sentences. In order to minimize potential tonal coarticulations caused by the following syllable, all stimuli were placed sententence-finally. In addition to the 38 stimulus sentences ( 2 for each minimal pair), 62 fillers were also included. The sentences were semi-randomized on the list and presented to the subjects. All sentences on the list were composed of 9-15 characters. Following are some of the examples of the testing sentences (1-2) and fillers (3).

(1).Hen3 duo1 ren2 meng4xiang3 yi4 you2 balli2.

Many people dream of visiting Paris.

(2). Wuo3men li4 yong4 jia4ri4 qu4le balli3.

We went to Bali ${ }^{1}$ on the holiday.

(3).Can1jial yan3chang4hui4 shi2 bu2yao4 qing2xu4 shilkong4.

Do not lose the control of your emotion when attending a concert.

\subsection{Equipment}

Recordings were conducted by a CENIX mini digital recorder.

The data were acoustically analyzed by the software Praat.

\subsection{Procedure}

Subjects were instructed to operate the mini digital recorder so that they could self-pace the recording. A brief testing session was conducted to confirm the subjects' capability of correctly operating the recorder, and to familiarize the subjects with the format of the sentences on the list. All the sentences were recorded in one session without any breaks.

\subsection{Measurements}

The pitch contour of each syllable was first manually classified as dipping, falling, and level. A Praat script was then written for automatic pitch extraction of each contour type; pitch tracking were later hand-checked and -corrected. In the dipping contour, three reference points were extracted using a Praat script - the initial high (onset), the medial low (turning point), and the final high (offset). In the falling and level contour, only the onset and the offset were measured. Should the pitch difference between

\footnotetext{
1 A suburban area in Taipei, Taiwan.
} 
the onset and the offset be lower than $5 \mathrm{~Hz}$, this study categorizes the contour as level.

\section{RESULTS}

\subsection{Tonal Contour}

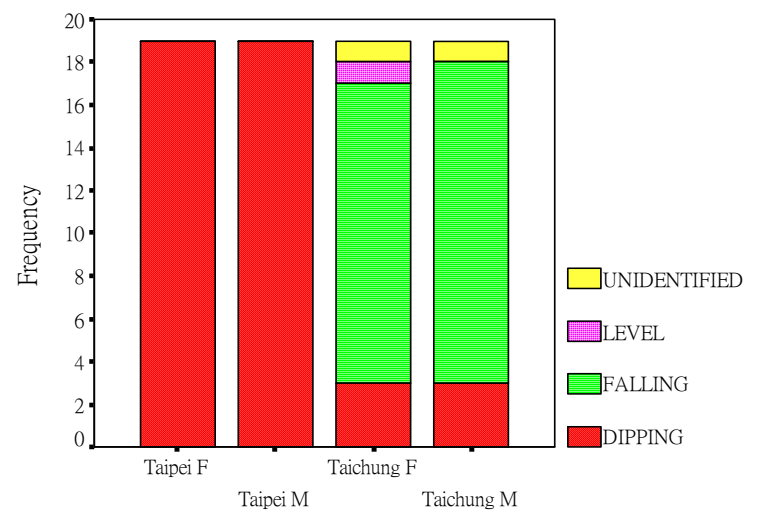

Fi

g.1 Frequencies of Various T2 Contours

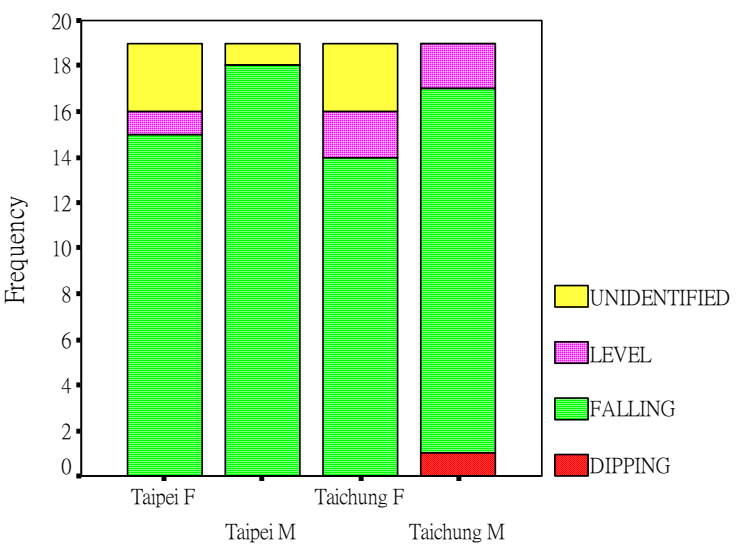

$\mathrm{Fi}$

\section{g. 2 Frequencies OF Various T3 Contours}

As shown in Figures 1 and 2, the discrepancy of T2/T3 contours between Taipei and Taichung is apparent. In Taipei, the majority of $\mathrm{T} 2$ and $\mathrm{T} 3$ were realized as dipping and falling tones respectively. Contrastively, the majority of both T2 and T3 were realized as falling tones in Taichung.

\subsection{Regional Discrepancy}

The tonal contour discrepancies mentioned in 3.1 indicate the regional discrepancy of T2 and T3 in Taiwan. Statistical analyses showed significant results on the ending points of $\mathrm{T}^{2}$ and $\mathrm{T} 3$, further implying the regional discrepancy. $(\mathrm{T} 2: \mathrm{F}(1,24)=21.716$, $\mathrm{p}<.05 ; \mathrm{T} 3: \mathrm{F}(1,24)=8.277, \mathrm{p}<.05)$

\subsection{Merger of $\mathrm{T} 2$ and $\mathrm{T} 3$}

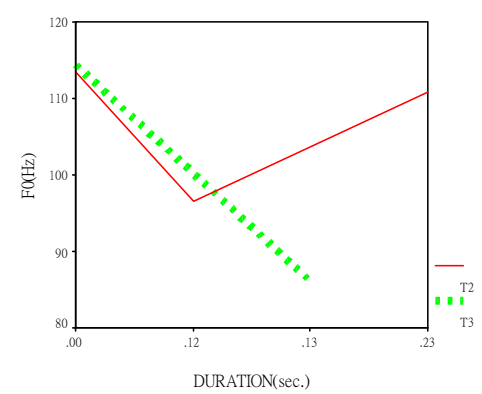

Fig.3 T2 and T3 Contours of the Taipei Male

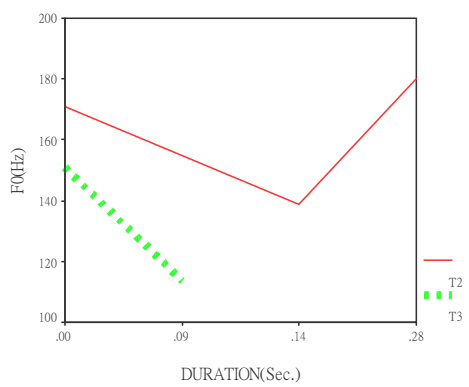

Fig.4 T2 and T3 Contours of the Taipei Female

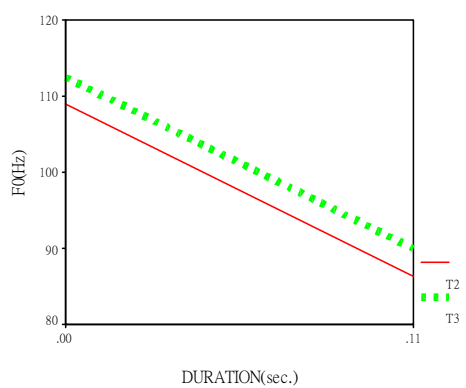

Fig.5 T2 and T3 Contours of the Taichung Male

\footnotetext{
${ }^{2}$ For Taipei T2, the mid point was adopted to compare to the Taichung $\mathrm{T} 2$ ending point.
} 


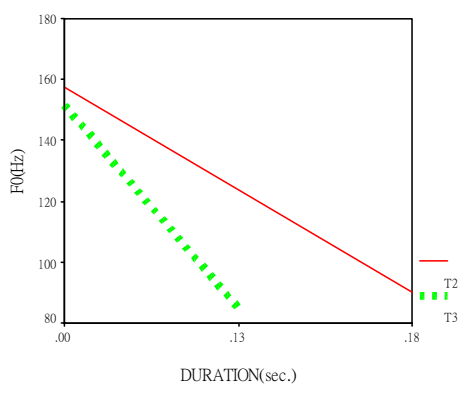

Fig.6 T2 and T3 Contours of the Taichung Female

As indicated in 3.1, Taichung speakers tended to pronounce both $\mathrm{T} 2$ and T3 as falling tones In order to investigate whether the falling T2 and the falling T3 have been merged in Taichung, statistical analyses were applied on the beginning and ending points of $\mathrm{T} 2$ and $\mathrm{T} 3$. Results showed that none of the comparisons were significant in Taichung, indicating an emerging merger in this area (Figures 3-6) )(Begin: $F(1,24)$ $=.25$, ns.; (End: $F(1,24)=.05, \mathrm{~ns})$.

\section{DISCUSSION}

This study observes the appearance of regional varieties of Guoyu. The controversial language policy of Guoyu in early days has created a dichotomy of the stigmatized "Taiwan Guoyu", i.e., the phonologically Taiwan Min affected Guoyu, and the prestigious "standard Guoyu". This policy did successfully decrease the degree of the stigmatized Taiwan Guoyu. However, the "remaining" Guoyu, although considered as "standard", can never be maintained homogeneous. Varieties of regional, social class, or other dimensions, are thus likely to appear.

Of course, due to its small scale, one can merely consider the current results as those of a pilot study. However, as the Guoyu tonal system and its regional varieties have hitherto barely been researched, such a pilot study presents a preliminary trial and a beginning for further research.

\section{ACKNOWLEDGEMENTS}

This study could not have been possible if it were not for my advisor, Prof. Janice Fon. Naturally, all the faults are mine.

\section{REFERENCES}

[1] Chao, Yuen-ren. A Grammar of Spoken Chinese. Berkeley \& Los Angeles: A grammar of spoken Chinese, 1968.

[2] Fon, Janice \& Huiju Hsu. "Positional and Phonetic Effects on the Realization of Taiwan Mandarin Tone 2." Paper presented at the Interspeech 2004, Jeju, Korea 2004.

[3] Fon, Janice, and Wen-yu Chiang. "What Does Chao Have to Say About Tones? -- a Case Study of Taiwan Mandarin." Journal of Chinese Linguistics 27, no. 1 (1999): 13-37.

[4] Hsu, Hui-ju. "Language Shift in Taiwan -- a Case Study." Paper presented at the SALSA VI, Austin, Texas 1998.

[5] Kubler, Cornelius. The Development of Mandarin in Taiwan: A Case Study of Language Contact. Taipei: Student, 1985.

[6] Lo, Chao-chin. "nau4 re4 gun3gun3-da4zhong4 chuan2bo4 de fen1he2" The World of Chinese Language and Literature 7, no. 6 (1991): 12-15.

[7] Milroy, James. Linguistic Variation and Change. Oxford: Blackwell, 1992.

[8] Tzeng, Chin-chin. "A Contrastive Analysis on the Pronunciation between Mainland China and Taiwan: A Case Study of News Reporters." Taipei: NSC 88-2411-H-003-017, 1999. 